Article

\title{
A Nine-Year Climatology of Arctic Sea Ice Lead Orientation and Frequency from AMSR-E
}

\section{David Bröhan * and Lars Kaleschke}

Institut für Meereskunde, University of Hamburg, Bundesstraße 53, D-20146 Hamburg, Germany; E-Mail: Lars.Kaleschke@ zmaw.de

* Author to whom correspondence should be addressed; E-Mail: David.Broehan@zmaw.de; Tel.: +49-40-42838-5430; Fax: +49-40-42838-7471.

Received: 17 December 2013; in revised form: 31 January 2014 / Accepted: 10 February 2014 / Published: 18 February 2014

\begin{abstract}
We infer the fractional coverage of sea ice leads (as concentration) in the Arctic from Advanced Microwave Scanning Radiometer for Earth Observing System (EOS) (AMSR-E) brightness temperatures. The lead concentration resolves leads of at least $3 \mathrm{~km}$ in width. We introduce a new algorithm based on the progressive probabilistic Hough transform to automatically infer lead positions and orientations from daily AMSR-E satellite observations. Because the progressive probabilistic Hough transform often detects an identical lead several times the algorithm clusters neighboring leads that belong to one lead position. A first comparison of automatically detected lead positions and orientations with manually detected lead positions and orientations reveals that $57 \%$ of the reference leads are correctly determined. Around $11 \%$ of automatically detected leads are located where no reference lead occurs. The automatically detected lead orientations are distributed slightly differently from the reference lead orientations. A second comparison of automatically detected leads in the Fram Strait to leads in a wide swath mode Advanced Synthetic Aperture Radar scene shows a good agreement. We provide an Arctic-wide time series of lead orientations for winters from 2002 to 2011. For example, while a lead orientation of $110^{\circ}$ with respect to the Greenwich meridian prevails in the Fram Strait, lead orientations in the Beaufort Sea are more isotropically distributed. We find significant preferred lead orientations almost everywhere in the Arctic Ocean when averaged over the entire AMSR-E time series.
\end{abstract}

Keywords: sea ice; remote-sensing; leads; lead orientation; image analysis 


\section{Introduction}

In the context of sea ice, the World Meteorological Organisation (WMO) defines a lead as a more than $50 \mathrm{~m}$ wide rectilinear or wedge-shaped crack from several kilometers to hundreds of kilometers in length in the ice cover. The term lead is often used more generally as a synonym for a linear kinematic feature which describes different types of openings in the Arctic sea ice cover like Polynya, crack, fracture and failure zone [1,2]. The lead orientation both reflects and influences sea ice dynamics [3]. Although the lead orientation contains information about how sea ice is impacted by the surface wind, ocean currents, and internal forces in the sea ice, only a limited observational record exists. The scope of this study is to provide a time series of lead orientation for the lifespan of Advanced Microwave Scanning Radiometer for EOS from 2002 to 2011. Furthermore, we introduce for the first time a fully automatic method to derive lead orientations.

While some studies [3,4] and ongoing research focus on the distribution of leads, continuous observation of lead orientation still remains challenging. Inferring lead orientation on an Arctic basin-wide scale via remote sensing is not straightforward although observations of lead occurrence can be obtained from optical sensors, altimeters, and synthetic aperture radar. All of these remote sensing approaches face limitations when it comes to inferring the lead orientation. Optical sensors usually have a higher resolution than passive microwave sensors but they are often limited by cloud conditions. Altimeter track profiles alone are not sufficient to estimate the lead orientation. Synthetic aperture radar has a high spatial resolution but the temporal coverage is mostly not continuous. Furthermore, the ambiguity between multi-year ice and frost flowers in lead signatures limits the classification of leads from synthetic aperture radar [5]. We choose AMSR-E measurements as input for our approach although certain sensors offer a higher spatial resolution. AMSR-E offers a moderate spatial resolution combined with a continuous daily coverage of the entire Arctic.

Miles and Barry [4] detect leads in images of the western Arctic for winters from 1979 to 1985 recorded by the operational line scanning (OLS) sensor on the Defense Meteorological Satellite Program (DMSP). They manually inferred the lead orientation by drawing a line through a lead visible in OLS images. The manual approach is still the best option to detect reference leads for an evaluation of automatically detected leads. To be less susceptible to human perception and to reduce the manual workload, we here use an automatic approach for inferring the lead orientation based on the Hough transform.

This Hough transform is an image analysis technique to detect line structures like leads in images. Fetterer and Holyer [6] were the first to apply the Hough transform in a semi-automatic approach to detect leads from Advanced Very High Resolution Radiometer (AVHRR) satellite images. Lindsay and Rothrock [3] list some alternative approaches to the Hough transform: the local gradient in an image (Sobel operator), 2D-Fourier transform, skeleton orientations [7], and the direction of maximum extent. Lindsay and Rothrock [3] performed a semi-automatic detection of lead orientations from AVHRR images for the year 1989. Their method recognizes also small scale leads, but covers only small parts of the Arctic. Lindsay and Rothrock [3] rejected the Hough transform, because the Hough transform was at that time subject to rather arbitrary choices. While Lindsay and Rothrock [3] decided to develop a semi-automatic method based on the maximum extent of a lead, we apply a more recent and 
a more advanced version of the Hough transform, the progressive probabilistic Hough transform by Galambos et al. [8]. This version of the Hough transform provides additional input parameters in comparison to the classical Hough transform. Furthermore, the progressive probabilistic Hough transform analyzes only a subset of image pixels which reduces the calculation time.

There have been no published attempts after Fetterer and Holyer [6] to infer lead orientations in an automatic manner using the Hough transform. We seek to automatically infer lead orientations from passive microwave sensors. This allows us to derive a climatology of automatically-obtained lead positions and orientations that can be prolonged into the future by applying our algorithm to Advanced Microwave Scanning Radiometer 2 (AMSR2) observations.

The starting point of our study are Arctic-wide maps of lead concentration with a grid resolution of $6.25 \mathrm{~km}$. We estimate the lead concentration by taking the emissivity ratio between $19 \mathrm{GHz}$ and 89 $\mathrm{GHz}$ brightness temperature channels of the passive microwave sensor AMSR-E and applying an edge detecting filter on this ratio [9]. The lead concentration algorithm provides no directional information. We detect line structures within the lead concentration maps from which an orientation can be inferred.

A possible implication of this study are that the derived lead orientations can be used to evaluate lead-resolving sea ice models. A local structure tensor in a sea ice model as a measure of the sub-continuum anisotropy (such as leads) of the sea ice cover results in substantially changed sea ice thickness and drift in comparison to a reference run [10]. Girard et al. [11] conclude that the poor representation of observed sea ice deformation patterns that represent lead structures in two high-resolution sea ice models could mainly be addressed to the used mechanical framework. The build-up of leads and the consequent anisotropy of the sea ice deformation can be captured within an elasto-brittle or an elastic-decohesive framework [12-15].

This paper is organized as follows: We start with a short description of the AMSR-E satellite sensor in Section 2. In Section 3, we introduce the Hough transform and an algorithm that clusters multiple detected leads belonging to an identical lead position. We evaluate the automatically detected leads with manually detected leads and compare them to Advanced Synthetic Aperture Radar (ASAR) images in Section 4. First results of Arctic-wide lead distributions are presented in Section 5. We discuss our results in Section 6 and conclude them in Section 7.

\section{Data}

We estimate the lead concentration from AMSR-E passive microwave data following an approach developed by Röhrs et al. [9]. Japan Aerospace Exploration Agency (JAXA) AMSR-E sensor is mounted on the National Aeronautics and Space Administration (NASA) EOS Aqua satellite. The satellite was launched in 2002 and provided measurements until 2011. AMSR-E measures at six different frequencies ranging from approximately $19 \mathrm{GHz}$ to $89 \mathrm{GHz}$. We use the level-3 gridded product of the $19 \mathrm{GHz}$ [16] and $89 \mathrm{GHz}$ [17] vertically polarized brightness temperature channels with a pixel resolution of $6.25 \mathrm{~km}$ times $6.25 \mathrm{~km}$. Like Röhrs et al. [9], we focus on leads in the closed sea ice cover. Thus, pixels are not considered that contain more than $10 \%$ of open water according to (Arctic Radiation and Turbulence Interaction STudy (ARTIST) Sea Ice (ASI) concentration [18,19]. 
We use Advanced Synthetic Aperture Radar (ASAR) wide swath mode (WSM) images to validate our automatically detected lead orientations with independent measurements. ASAR provides C-band measurements at a frequency of $5.25 \mathrm{GHz}$ as normalized radar backscatter coefficients, which depict partly the surface roughness. ASAR WSM images are processed to a $150 \mathrm{~m}$ resolution. The swath width is approximately $400 \mathrm{~km}$. The ASAR sensor is mounted on European Space Agency (ESA)'s satellite Envisat. ESA provides ASAR observations until April 2012 when contact to Envisat was lost.

\section{Methods}

In this section, we briefly present the algorithm by Röhrs et al. [9] to infer the lead concentration (Section 3.1). The core of the new algorithm is the Hough transform (Section 3.2) together with a cluster algorithm (Section 3.3). In Section 3.4, we introduce the C-score to evaluate a detected lead with the underlying lead concentration. We connect the individual pieces in an outline of the entire algorithm in Section 3.5.

\subsection{Lead Concentration}

When sea water freezes in winter, nilas is formed as the first consolidated type of thin ice. Röhrs et al. [9] found that nilas shows a unique signature in the emissivity ratio between the $19 \mathrm{GHz}$ and $89 \mathrm{GHz}$ vertically polarized brightness temperature channels. We estimate the lead concentration by calculating the emissivity ratio between the two AMSR-E brightness temperature channels. In order to highlight lead structures, Röhrs et al. [9] applied a spatial median filter on the brightness temperature ratio $r$. We apply the same brightness temperature ratio tie points $\left(r_{0 \%}=0.015\right.$ and $\left.r_{100 \%}=0.05\right)$ as Röhrs et al. [9] to estimate the lead concentration [20]. The algorithm detects leads having a width of at least $3 \mathrm{~km}$ in the sea ice cover although the grid resolution of the lead concentration covers $6.25 \mathrm{~km} \times 6.25 \mathrm{~km}$. AMSR-E resolves the radiometric signal of a lead fraction approximately greater than $50 \%$ in a grid cell. Leads smaller than $3 \mathrm{~km}$ in width are sometimes depicted as fragments visible as pixels showing no distinct structure. In order to reduce the number of fragments, we remove single pixels in the binary lead concentration maps with a morphological hit-or-miss transform. The binary lead concentration consists of two classes: 0 for $0 \%$ lead concentration and 1 for $1 \%$ to $100 \%$ lead concentration. From a lead concentration map one can manually determine the lead orientation by drawing a line through a lead structure and estimating the orientation of this line. We apply this manual approach in order to obtain reference leads for a comparison with automatically detected leads. The automatic detection of lead orientations is explained in the following sections.

\subsection{Hough Transform}

The principle of the Hough transform is shown in Figure 1. Paul V.C. Hough originally invented the Hough transform in 1962 to recognize complex lines in photographs or other pictorial representations [21]. Duda and Hart [22] applied the Hough transform to picture analysis. With the Hough transform we seek to answer the question: Which line connects points that are stringed together? Due to unresolved lead fragments the lead concentration shows a lot of none line-like 
patterns. The purpose of the Hough transform is to discern lead structures with a line shape from other geometrical fragments.

Figure 1. Principle of the Hough transform: An arbitrary point $\left(x^{\prime}, y^{\prime}\right)$ in a coordinate system is intersected by lines with arbitrary slopes $m$ and y-offsets $b(\mathbf{a})$. All lines are described by one parameter line (b). The parameter lines (c) belong to a chain of corresponding points (d). The points are connected via a line with the slope $m^{\prime}$ and the y-intersect $b^{\prime}$ indicated by a red arrow.
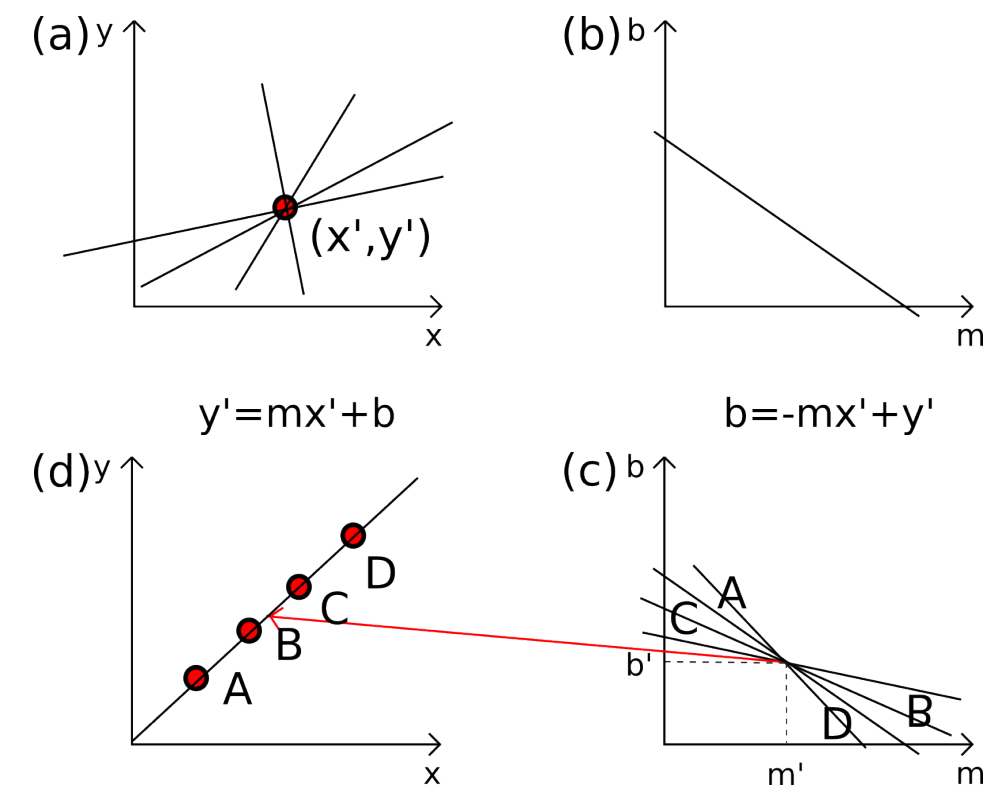

Figure 1a shows a point in a coordinate system. The point is intersected by lines with all possibles slopes $m$ and y-intersects $b$ :

$$
y^{\prime}=m x^{\prime}+b
$$

Equation (1) can be rewritten to

$$
b=-m x^{\prime}+y^{\prime}
$$

representing all lines in the normal space that intersect in the point $\left(x^{\prime}, y^{\prime}\right)$ as one parameter line in the parameter $(m, b)$ space (Figure $1 b)$. Figure 1d shows a chain of unconnected points. The corresponding parameter lines are shown in Figure 1c. In our example, four entries accumulate where the parameter lines intersect. Therefore, the space is named accumulator space. We determine the line that connects the points A, B, C, and D in Figure 1d with the slope $m^{\prime}$ and the y-intersect $b^{\prime}$ at the intersection of the four parameter lines inserted in Equation (1).

Because if the slope $m$ reaches zero the linear formulation will fail, the normal parametrization of a line is often chosen for the Hough transform:

$$
l=x \cos (\theta)+y \sin (\theta)
$$

$l$ - normal line distance from the origin

$\theta$ - angle between distance and the $\mathrm{x}$-axis

$x, y-\mathrm{x}, \mathrm{y}$ - plane coordinates. 
We use an alternative implementation of the Hough transform, the progressive probabilistic Hough transform by Galambos et al. [8]. The major difference to the classical Hough transform is that the progressive probabilistic Hough transform uses only a randomly selected sample of image pixels. The progressive probabilistic Hough transform offers three input parameters:

1. Threshold value - pixels with a lower number of occurrences in the accumulator space are not considered as a lead. For every randomly selected point, the progressive probabilistic Hough transform tests the hypothesis if the number of occurrences in the accumulator space could be due to random noise. The classical Hough transform also uses a threshold value as input, but the classical Hough transform calculates the number of occurrences for every pixel and not only for a sample of pixels.

2. Minimum line length—leads shorter than this length are neglected. A minimal line length reduces the computing time significantly [8].

3. Maximum line gap—distance (in pixels) the progressive probabilistic Hough transform is allowed to fit when a lead is interrupted.

One major advantage of the progressive probabilistic Hough transform over the classical Hough transform is that the progressive probabilistic Hough transform detects finite lines. A detailed algorithm outline of the progressive probabilistic Hough transform can be found in Galambos et al. [8]. We refer to the term probabilistic progressive Hough transform henceforth with the term Hough transform.

\subsection{Cluster Algorithm}

Because the Hough transform often detects identical lead positions several times we cluster lead detections that are located around only one lead position in a lead concentration map (Figure 2). The criterion is the maximal euclidean distance $d$ a cluster is allowed to have to a neighboring cluster. In order to illustrate the functionality of the cluster algorithm, we create an idealized test data set consisting of six leads (Figure 3a).

The cluster algorithm uses a proximity matrix (Figure 3b) to evaluate the criterion of for example $d=2$ pixels. The matrix elements consist of distances between the lead centers. Only distances smaller than $d=2$ pixels are shown. Distances smaller than $d=2$ pixels indicate that two leads belong to the same cluster. By looking at the rows and columns of the matrix, we see that lead center 3 misses a lead center within the distance $d=2$. Lead centers 4 and 5 build a cluster. The remaining lead centers 1, 2, and 6 build the last cluster. Lead centers 2 and 6 are clustered indirectly via the remaining lead center 1 .

We apply the developed cluster algorithm with a maximal distance $d=4$ pixels to the leads detected by the Hough transform. The cluster algorithm greatly reduces the number of leads like for an example north of Greenland on 14 March 2011 (Figure 2). In this example the cluster algorithm reduces ten leads to four clustered leads.

We tested maximal distances ranging from $d=2$ to $d=7$ in varying sub-regions of the Arctic on different days. We conclude that if we increase the maximal distance the total number of leads will further decrease, but we run a higher risk to cluster leads that do not belong to the same lead. With the subjective choice of a rather small maximal distance $d=4$ pixels, we accept that some leads are still 
detected several times (Figure 2). Otherwise, no lead was misplaced by the clustering in the evaluated examples with a maximal distance $d=4$ pixels.

Figure 2. Comparison between unclustered leads (red lines) and clustered leads (yellow lines) in the Fram Strait and a regional zoom north of Greenland on 14 March 2011. The lead concentration is depicted in blueish colors. The cluster distance $d$ equals 4 pixels or $25 \mathrm{~km}$.

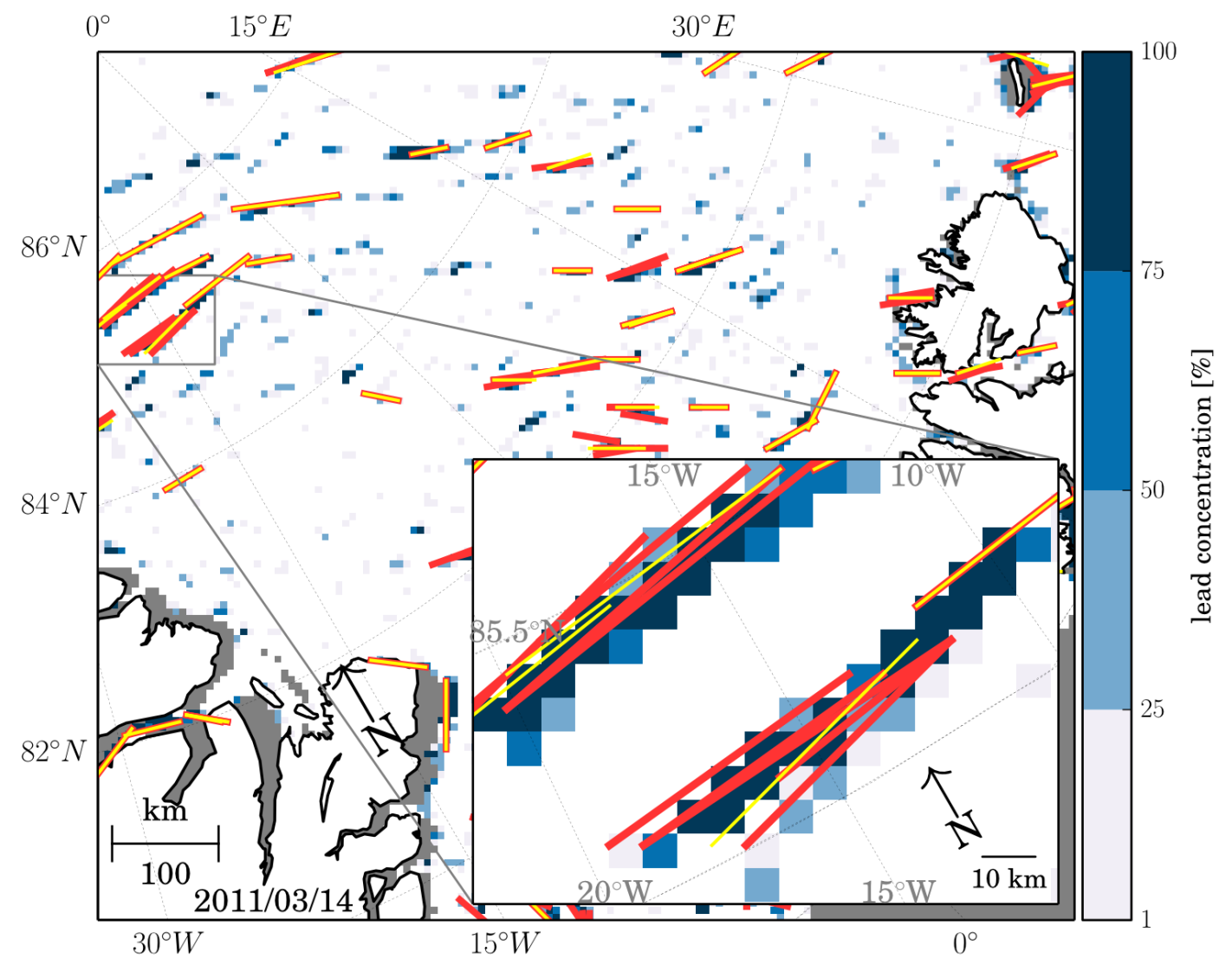

Figure 3. Principle of the cluster algorithm: Leads (black dotted lines) are reduced to lead centers (black cross, abbreviated to L) in a test data set (a). The numbers in the proximity matrix (b) represent the distance between the lead centers. All distances greater than the maximal distance $d=2$ are whitened. Intersecting rows and columns with an entry identify lead clusters belonging to one cluster. The clustered lead centers are depicted as gray squares and the clustered leads as gray lines (a).
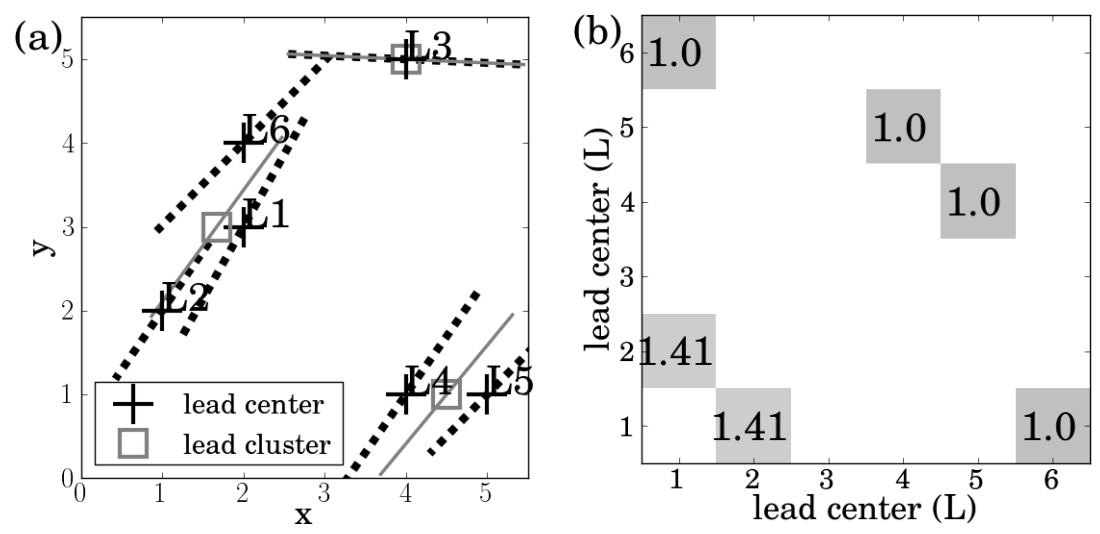


\subsection{C-Score}

In order to check if for example a lead is misplaced by the clustering, we evaluate the lead via the consistency $(C)$ or C-score:

$$
\mathrm{C}=\frac{\text { lead concentration pixels }}{\text { Hough pixels }}
$$

The C-score checks if pixels covered with a lead detected by the Hough transform is underlaid by lead concentration pixels. The more pixels of a lead detected by the Hough transform are covered with pixels of lead concentration, the closer the C-score gets to 1 .

\subsection{Algorithm Outline}

In the end of this section, we bring all components comprising the algorithm to infer the lead orientation together in an outline of the algorithm. The Hough transform needs a threshold value and a minimal line length as input parameters in order to detect leads. We keep the maximum line gap (Section 3.2) fixed to one pixel. We test several threshold parameter and minimal line length combinations and conclude that one combination that is most accurate for every day does not exist.

Because one combination of input parameters does not exist we use a set of varying input parameter combinations for every day. We evaluate the quality of a input parameter combination for the Hough transform via the daily mean C-score (Section 3.4). We test all possible Hough transform input combinations for threshold values between 5 and 50 and minimal line lengths between 5 and 15 pixels. We select 50 Hough input parameter combinations out of 506 options that are closest to $C=1$ for a test period covering the whole Arctic and lasting from February to April 2011 ( $c f$. Table A1 in Appendix).

Figure 4 summarizes the steps of the complete algorithm. First, we apply an algorithm by Röhrs et al. [9] to obtain the lead concentration. Afterwards, we remove single pixels in the binary lead concentration maps with a morphological hit-or-miss transform. This step reduces the fragments in the image caused by unresolved small-scale leads. The Hough transform by Galambos et al. [8] is applied on the preprocessed binary image. We apply the most accurate 50 parameter combinations to every day of the AMSR-E time series and select the three most accurate parameter combinations via the C-score (Equation (4)). We select all leads with an individual C-score $C \geq 0.85$ detected by the Hough transform from the three most accurate parameter combinations. The resulting leads are clustered if the lead centers are closer than 4 pixels to one another. On these clustered leads, we apply the consistency criterion with $C \geq 0.5$ once again in order to remove leads that got heavily misplaced during the clustering. As the final step, we calculate the lead orientation as angle from the leads.

Figure 4. Sketch of the algorithm outline.

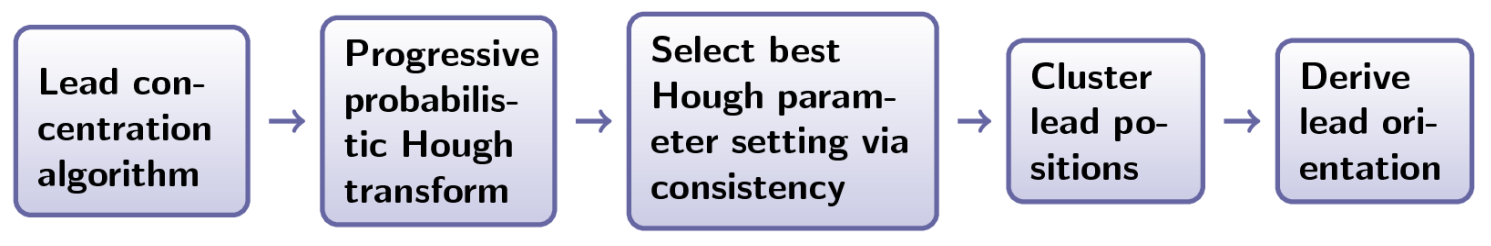




\section{Validation}

In this section, we evaluate leads detected by the Hough transform by using leads manually detected from a lead concentration map. Because we detect leads from daily lead concentration maps, we evaluate leads on the daily time-scale. The focus is in detail on the Fram Strait region on 14 March 2011 and the Beaufort Sea on 14 November 2004. We choose the Fram Strait and the Beaufort Sea, because lead patterns occur that are representative of the Arctic. Additionally, leads detected by the Hough transform are validated with leads visible in ASAR scenes.

\subsection{Evaluation of Leads Detected by the Hough Transform with Manually Detected Leads}

In order to compare manually detected reference leads with automatically detected leads, we zoom in the Fram Strait region on 14 March 2011 for a case study (Figure 5).

Figure 5. Comparison between reference leads (red) and clustered leads (yellow) detected by the Hough transform in the Fram Strait on 14 March 2011.

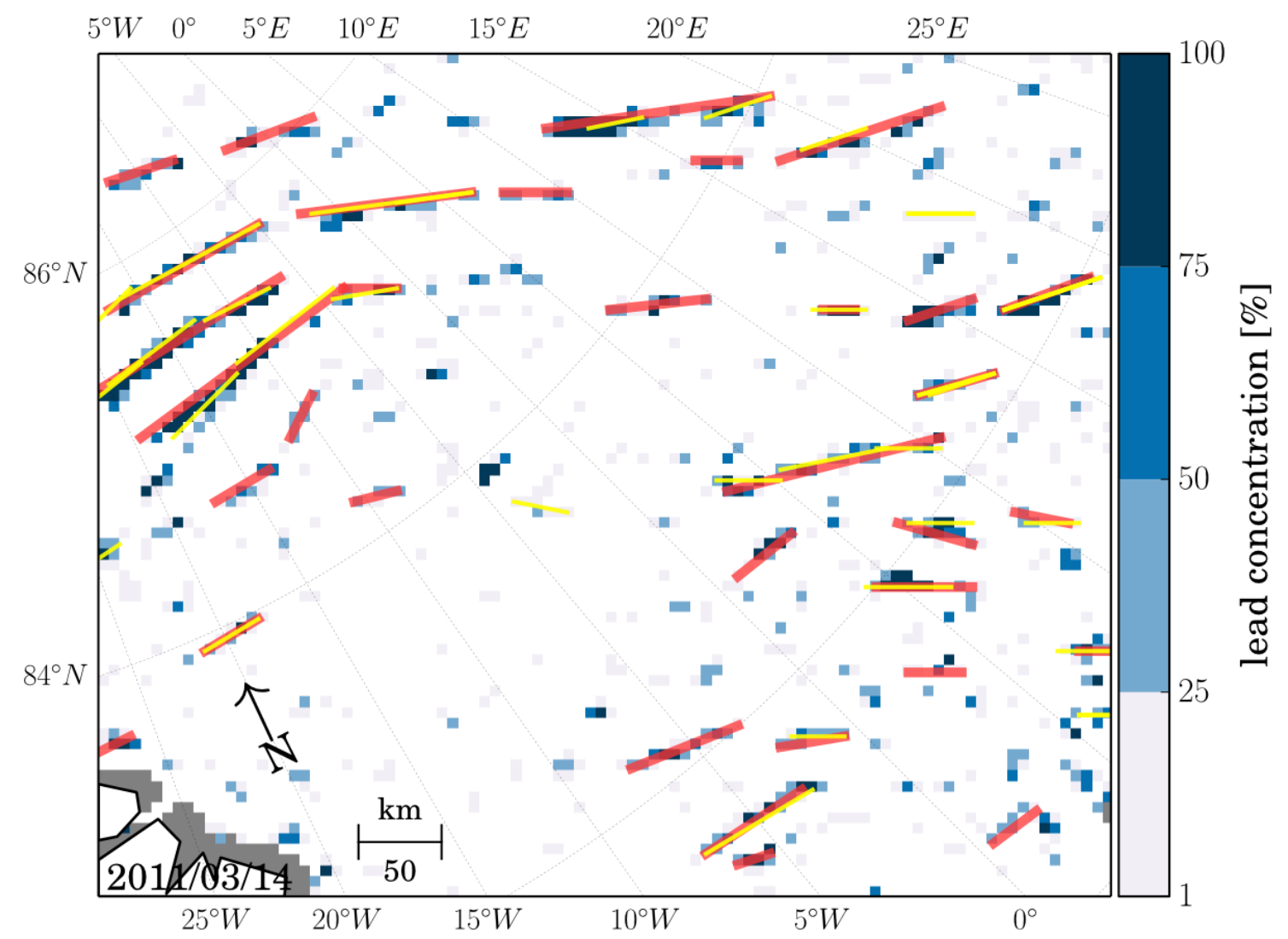

We analyze a similar map that is not shown here in the Beaufort Sea on 14 March 2011. To be less depending on the evaluation of only one day we extend the evaluation of the Beaufort Sea on 14 November 2004 (Figure 6).

We compared in total 155 reference leads to 173 clustered leads. On average, $57 \%$ of the reference leads are detected, $11 \%$ of the clustered leads are located where no reference lead occurs, and $17 \%$ of the reference leads are detected multiple times despite clustering (Table 1). In the next Section 4.2, we compare the lead distributions and test for a biased distribution of clustered leads. 
Figure 6. Comparison between reference leads (red) and clustered leads (yellow) detected by the Hough transform in the Beaufort Sea on 14 November 2004.

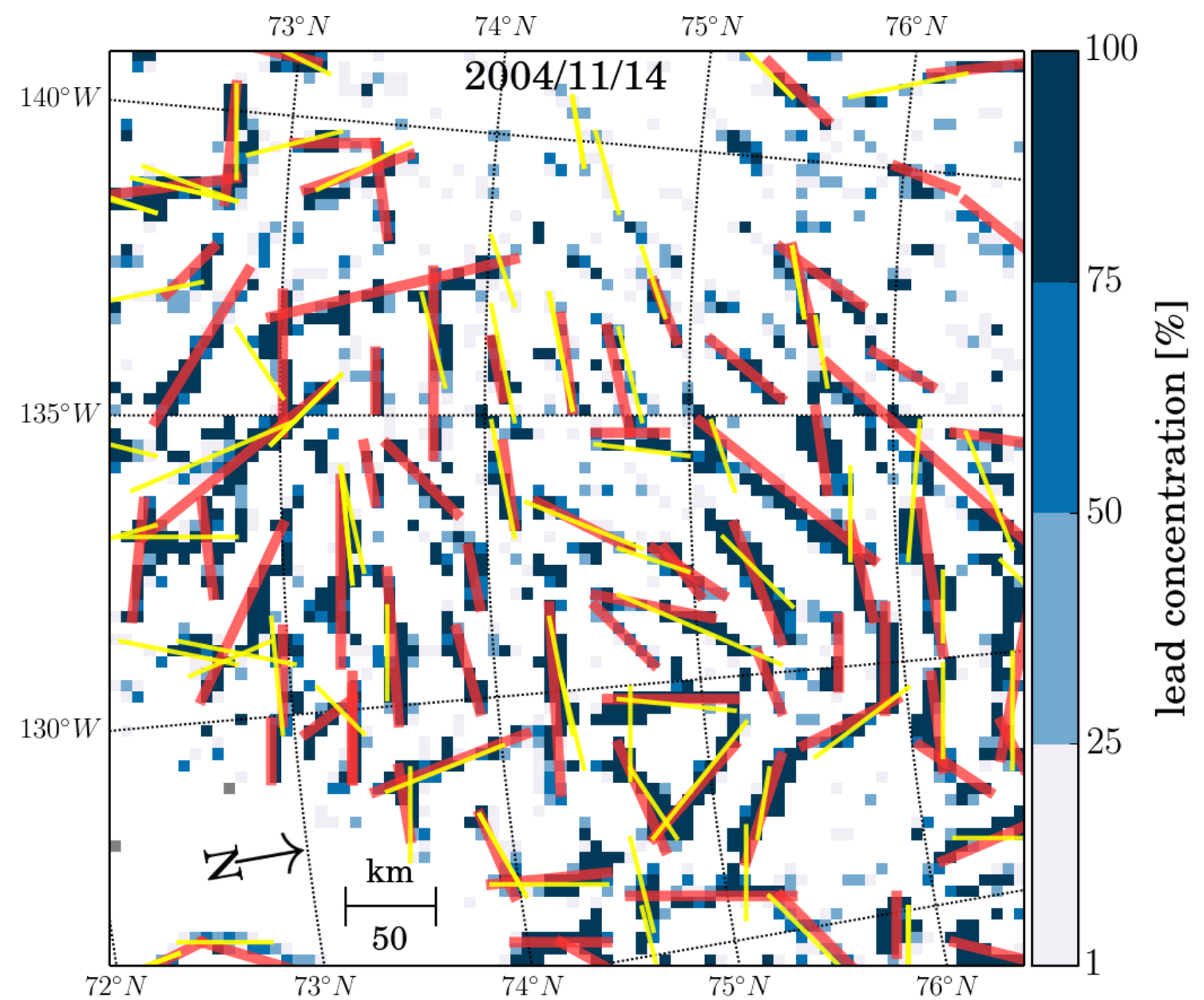

Table 1. Summary of a comparison of reference leads with leads detected by the Hough transform in three regions: Fram Strait (F) on 14 March 2011 (Figure 5), Beaufort Sea (B1) on the same day (map not shown), and Beaufort Sea (B2) on 14 November 2004 (Figure 6).

\begin{tabular}{lrrr}
\hline & \multicolumn{2}{c}{ Region } \\
& F & B1 & B2 \\
\hline $\begin{array}{l}\text { percentage [\%] of reference leads correctly detected by the Hough transform } \\
\text { percentage [\%] of leads detected by the Hough transform }\end{array}$ & 55 & 57 \\
that are located where no reference lead occurs & 10 & 9 & 13 \\
percentage [\%] of reference leads that are detected multiple times & 25 & 22 & 5 \\
average length [km] of a reference lead & 57 & 65 & 60 \\
average length [km] of a lead detected by the Hough transform & 48 & 50 & 53 \\
number of reference leads & 33 & 42 & 80 \\
number of leads detected by the Hough transform & 37 & 49 & 87 \\
\hline
\end{tabular}

\subsection{Distribution of Lead Orientations}

After the qualitative comparison, we apply basic statistics to answer the question: Does the distribution of reference leads differ from the distribution of leads detected by the Hough transform? 
Before we present the basic statistical parameters, we introduce the concept of lead orientations described by angles. The lead orientation is defined as the angle between the $0^{\circ}$ Greenwich meridian and the lead itself. A lead orientation of $0^{\circ}$ or $180^{\circ}$ is equal and is orientated parallel to the Greenwich meridian. The lead orientation is defined clockwise.

The need to calculate mean and standard deviation differently for circular data arises from the transition between $0^{\circ}$ to $180^{\circ}$. We estimate the angular mean orientation from a set of orientations after a common approach by Mardia and Jupp [23]:

$$
\begin{gathered}
\bar{X}=\sum_{i}^{N} \cos \left(2 \theta_{i}\right) \\
\bar{Y}=\sum_{i}^{N} \sin \left(2 \theta_{i}\right) \\
\bar{\theta}=\frac{\arctan (\bar{X}, \bar{Y})}{2}
\end{gathered}
$$

$$
\begin{aligned}
\bar{X}, \bar{Y} & - \text { unit vector components } \\
& \theta-\text { orientation angle between a lead and Greenwich meridian }
\end{aligned}
$$

Olson [24] showed that the four-quadrant arc tangent is line variant. Therefore, we multiply the lead orientations $\theta_{i}$, which range from $0^{\circ}$ to $180^{\circ}$, by 2 and divide the mean lead orientation $\bar{\theta}$ by 2 .

We choose to calculate the angular standard deviation comparably to the classical standard deviation for data on a line. The advantages are that the standard deviation is well known and provides results in the same dimension as the angular mean whereas the angular standard deviation is non-dimensional and ranges between 0 and 1 [4,23]. We can calculate the classical standard deviation, if lead orientations are distributed over a range smaller than $90^{\circ}$. If lead orientations are distributed over a range greater than $90^{\circ}$, we calculate the angular standard deviation by weighting the lead orientations around the angular mean that represents the center of the distribution. The source code of the algorithm written in the programming language python can be found in Listing 1 in Appendix. When we refer to mean or standard deviation, we refer henceforth to the angular mean or angular standard deviation.

The distribution of both, reference leads and leads detected by the Hough transform, show a similar shape in the histogram (Figure 7a). The mean values and standard deviations differ only by $2^{\circ}$. We use a bin width of $10^{\circ}$ although the number of bins $k$ should be estimated with Sturges' rule via $k=1+\frac{\log N}{\log 2}$ with $N$ being the number of measurements and log being the decimal logarithm [25]. For our example this would result in a number of bins $k=6$.

We calculate the Probability Density Function (PDF) for the two distributions with a kernel-density estimate (Figure 7b). We select Scott's rule as bandwidth estimator: $h=\sigma N^{-1 /(i+4)}$ with $h$ being the bandwidth, $\sigma$ being the kernel standard deviation, and $i$ being the number of dimensions [25]. In order to test if the two distributions origin from the same distribution we apply the two-sided Kolmogorov-Smirnov test. The two-sided Kolmogorov-Smirnov test works with the null hypothesis that two independent samples are drawn from the same continuous distribution. We cannot reject the null hypothesis at $25 \%$ or lower alpha values since the p-value of 0.21 almost equals $25 \%$. The 
Kolmogorov-Smirnov test indicates that the two distributions differ slightly although the PDFs obtained from a kernel density estimation differ when compared visually.

Figure 7. Comparison between lead orientations detected by the Hough transform with reference lead orientations in a histogram (a) and as probability density functions (b) in the Fram Strait on 14 March 2011. The Gauss curve in (b) is shown as reference.
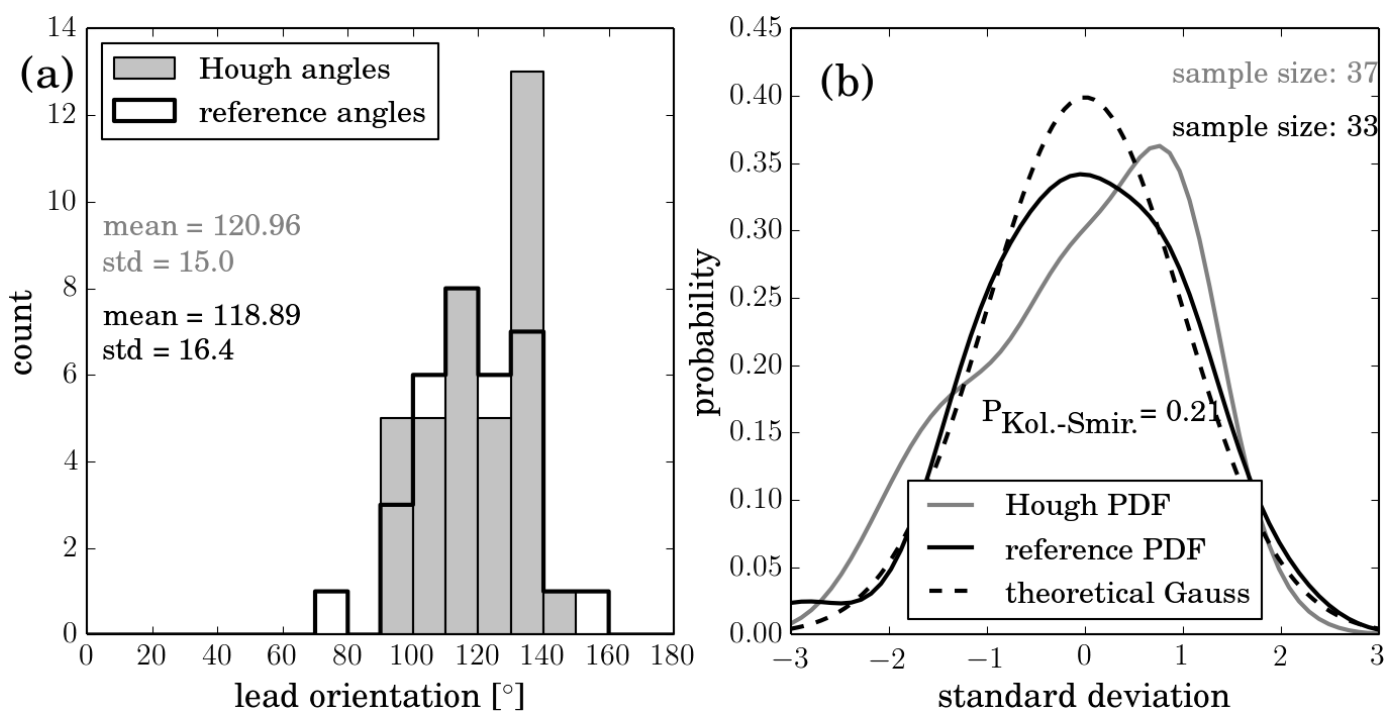

The mean orientation of the reference leads $\left(29^{\circ}\right)$ differs by $6^{\circ}$ from the leads detected by the Hough transform $\left(23^{\circ}\right)$ in the Beaufort Sea on 14 March 2011 (not shown). The standard deviation is $9^{\circ}$ smaller for leads detected by the Hough transform $\left(18^{\circ}\right)$. We cannot reject the null hypothesis at $17 \%$ or lower alpha values since the p-value is high (53\%). The Kolmogorov-Smirnov test indicates that the two distributions are samples from the same distribution.

Figure 8. Comparison between lead orientations detected by the Hough transform with reference lead orientations in a histogram (a) and as probability density functions (b) in the Beaufort Sea on 14 November 2004. The Gauss curve in (b) is shown as reference.
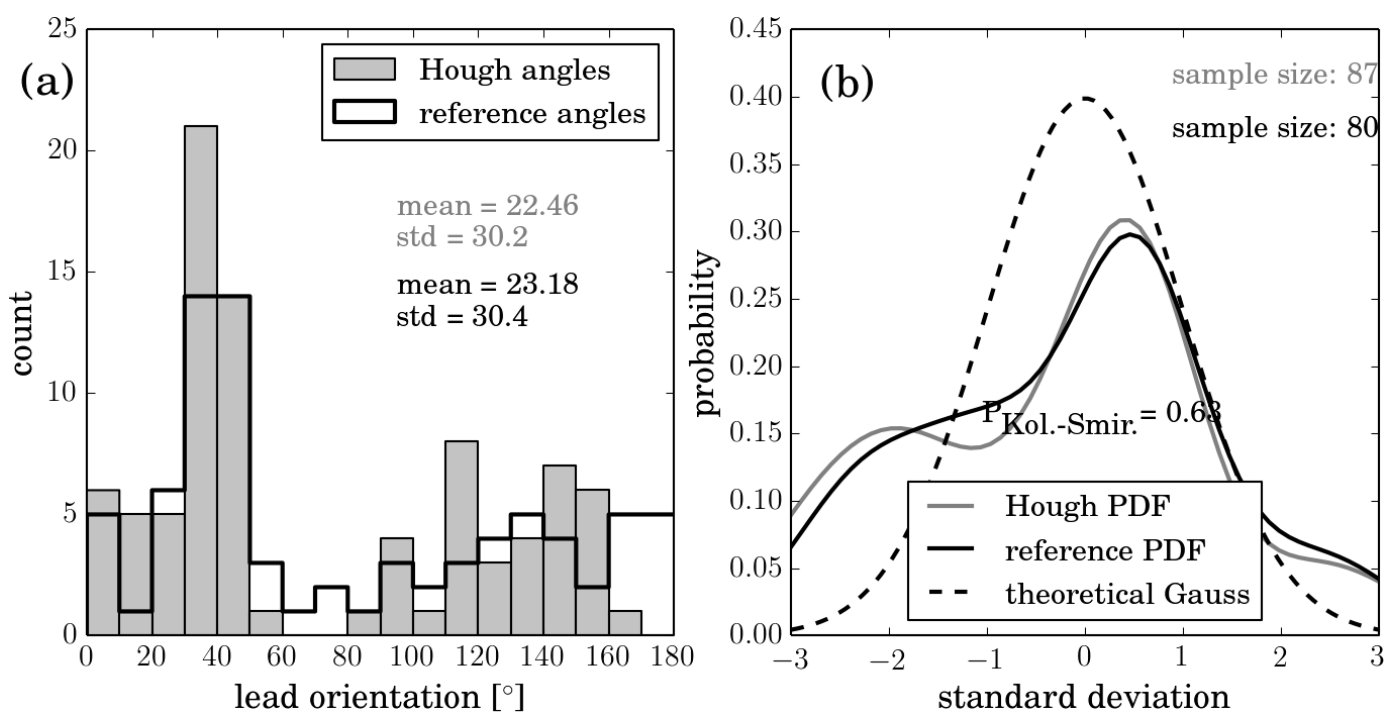
The distribution of both, reference leads and leads detected by the Hough transform, show a similar shape in the histogram of the Beaufort Sea on 14 November 2004 (Figure 8a). The mean values differ only by $1^{\circ}$, the standard deviations are identical. We cannot reject the null hypothesis at $11 \%$ or lower alpha values since the p-value is high (63\%). The Kolmogorov-Smirnov test indicates that the two distributions are samples from the same distribution. The shapes of the PDFs show also a similar pattern.

We observe a strong accordance for mean and standard deviation in all examples. The distributions of lead orientation differ slightly or look similar. Leads detected by the Hough transform do not show a biased distribution of lead orientations in comparison to a distribution of reference lead orientations. The differences in the PDFs in Figure 7b might indicate an influence of multiple detections of some leads by the Hough transform. Even though we do not detect every reference lead, we still obtain a sufficient sample size to be capable of determining the regional mean lead orientation.

Figure 9. Validation of clustered leads detected by the Hough transform with an ASAR scene shows a high accordance for larger leads. Leads (red lines) inferred from AMSR-E lead concentration (blueish pixels) are compared to ASAR observations north of Greenland (box in Figure 2) on 14 March 2011.

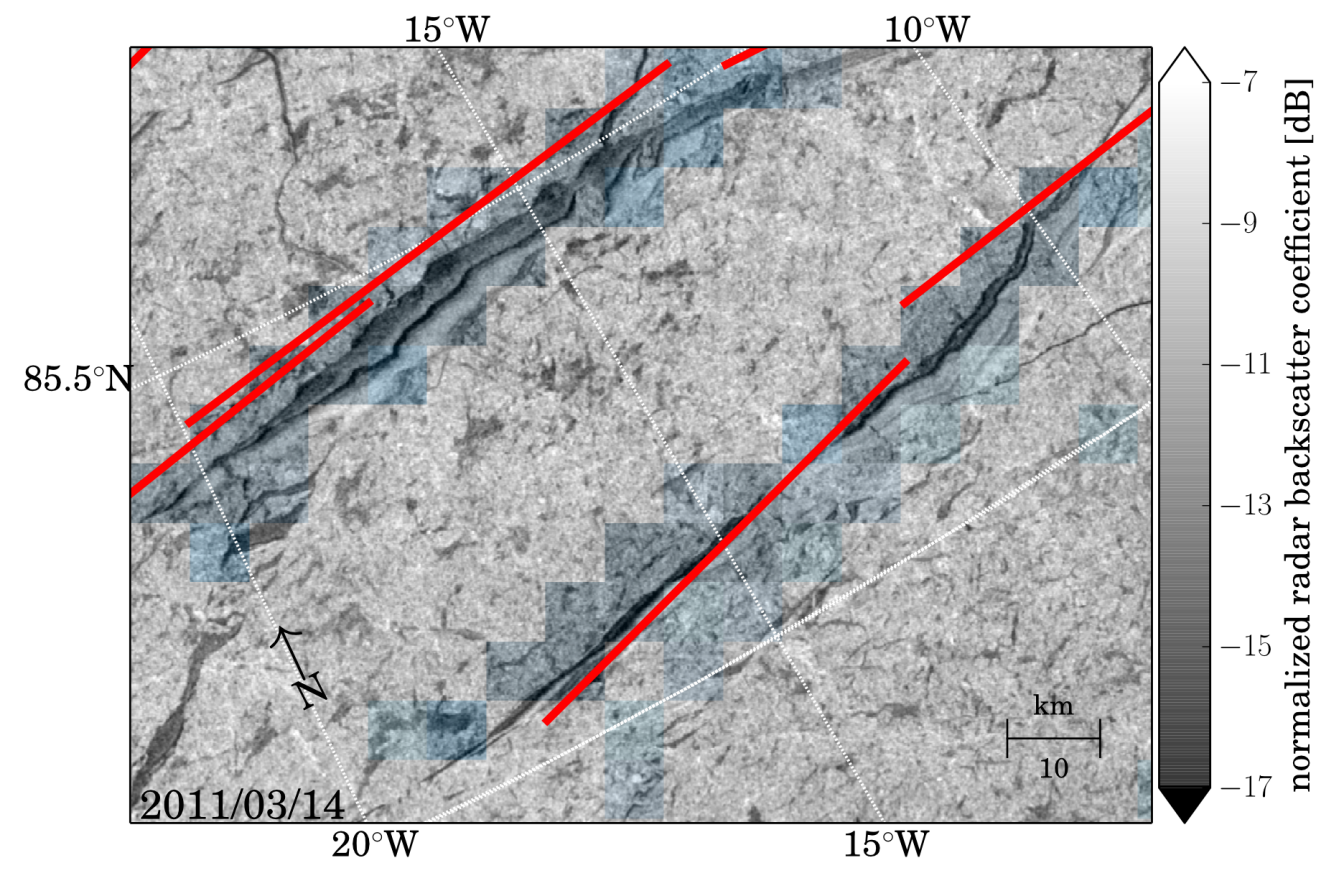

\subsection{Validation with ASAR}

We validate the leads detected by the Hough transform with ASAR images. Compared to AMSR-E, ASAR WSM scenes have a finer ground resolution $(\sim 70 \mathrm{~m} v s . \sim 6 \mathrm{~km})$. Accordingly, small leads and cracks, which are not resolved in an AMSR-E based lead concentration, are visible in an ASAR scene. We do not apply the Hough transform on ASAR scenes, because we choose to visually compare the orientation of automatically detected leads to leads in ASAR scenes. We compare 142 ASAR scenes to corresponding lead concentration maps and leads detected by the Hough transform from lead concentration maps. For consistency, we present an example in the Fram Strait on 14 March 2011 (Figure 9). Envisat ASAR scenes often cover the Fram Strait and the central Arctic. The Beaufort Sea is rarely covered by Envisat ASAR scenes. 
Leads detected by the Hough transform (red lines) are located close to large leads visible in the AMSR-E based lead concentration (blueish pixels) and in the ASAR scene (Figure 9). In the upper part of Figure 9, we observe a small displacement between the leads detected by the Hough transform and leads visible in the lead concentration and in the ASAR scene. The small displacement is caused by the clustering as a comparison with Figure 2 shows. For each of the two large leads visible in the ASAR image in Figure 9, still two automatically detected leads remain despite clustering. However, this is rather the exception when we compare with Figure 2 and 5. Multiple detections of leads probably do not cause a bias in the distribution of lead orientations ( $c f$. Section 4.2).

The quality of the lead concentration is substantial, because the Hough transform can only detect leads that are resolved by the AMSR-E based lead concentration. An extensive evaluation of the lead concentration can also be found in Röhrs et al. [9].

\section{Results}

We apply the Hough transform to derive the lead orientation for every day in winters from 2002 to 2011. In Section 5.1, we present a map showing the mean lead orientation from 2002 to 2011. Maps of monthly 9-year average lead orientations are shown in Section 5.2. We present the monthly variability of lead orientations in the Fram Strait and the Beaufort Sea in Section 5.3.

\subsection{Average Lead Orientation from 2002 to 2011}

After evaluating the automatically detected lead orientations with manually detected lead orientations and comparing the automatically detected leads to measurements of the independent ASAR sensor, we present the first results covering the entire time series from 2002 to 2011 . We show the spatial distribution of lead orientation in the Arctic (Figure 10). All lead observations from 2002 to 2011 are spatially averaged in cells of $100 \mathrm{~km}$ in length. The line orientation in a cell represents the mean lead orientation. Grey colors indicate the number of measurements - the darker, the more measurements. The line thickness depicts the standard deviation of all lead orientations in a cell. The standard deviation of random lead orientations in a cell equals roughly $55^{\circ}$. The pole hole is due to missing observations and due to some artificial pattern detected by the Hough transform at the edge of the pole hole which were cut out.

In order to identify cells in which the lead orientations are equally distributed from $0^{\circ}$ to $180^{\circ}$, we conduct a $\chi^{2}$-test within a Monte Carlo test for each cell. We apply the $\chi^{2}$-test for two distributions: the distribution of observed lead orientations detected by the Hough transform $\mathrm{H}_{\mathrm{obs}}$ in a cell and the distribution of random lead orientations $\mathrm{H}_{\text {rand }}$ from $0^{\circ}$ to $180^{\circ}$. We calculate the frequency of occurrence from the observed and random distributions. The $\chi^{2}$-test is applied 10.000 times to the observed as well as the random frequencies with changing random lead orientations for every individual $\chi^{2}$-test. The $\chi^{2}$-test checks the null hypothesis whether the observed lead orientations have the given frequencies of the random lead orientations. The $\chi^{2}$-test returns one probability for the null hypothesis not being rejected in each loop. The 10.000 individual probabilities are averaged to a mean probability $\bar{p}$. If the mean probability $\overline{\mathrm{p}}$ is smaller than 0.01 , the lead orientations in a cell will belong to a confidence level of $99 \%$ statistically significant non-random distribution, which is marked by a white dot in Figure 10. A 
prerequisite of the $\chi^{2}$-test is that at least five measurements must occur in each cell. With this test we do not check whether one, two, or several statistically significant mean lead orientation prevail. We check whether the lead orientations belong to a statistically significant non-random distribution.

Figure 10. Average lead orientation from 2002 to 2011. Grey colors depict the number of leads while the width of a lead measures the standard deviation of all lead orientations in a $100 \mathrm{~km} \times 100 \mathrm{~km}$ cell. White dots on top of a line indicate that the lead orientations are not randomly distributed on a confidence level of $99 \%$. The black dashed and gray squares mark sub regions which are going to be analyzed in Section 5.3.

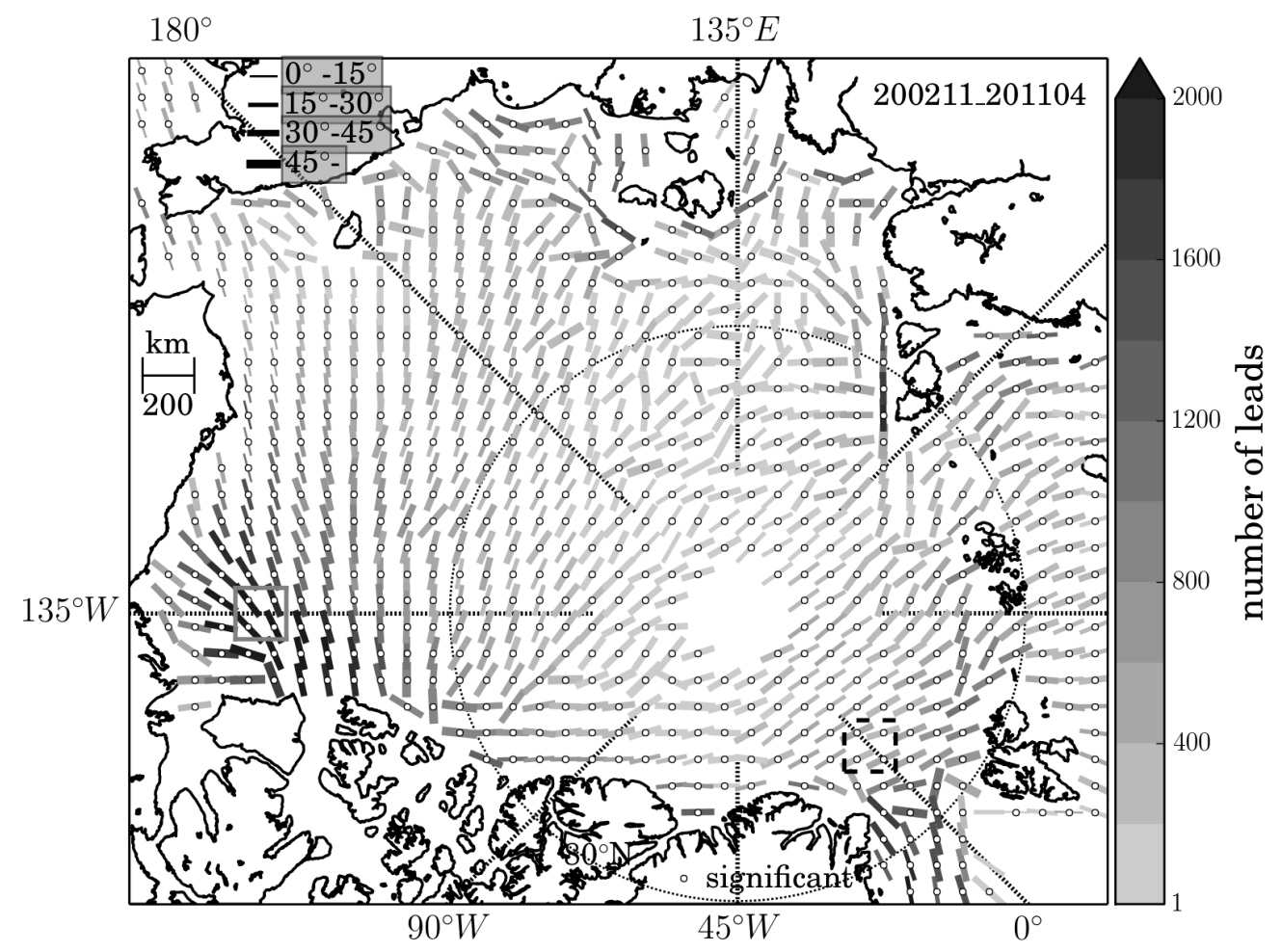

We observe some distinct pattern in Figure 10: Leads are often orientated coast-parallel except for some regions like the Canadian Archipelago near Alaska or the region north of Spitsbergen and Franz Josef Land. Most leads occur in the southern Beaufort Sea. Local maxima of lead occurrence are located north of Siberia and in the Fram Strait exit region.

The overall spatial pattern of the average lead orientation is rather smooth, spatial variations in lead orientation occur over larger distances. In the Fram Strait, leads are orientated parallel to a line drawn from northern Greenland to northern Spitsbergen. Lead orientation varies more in the Beaufort Sea: From almost being perpendicular to the coastline of Alaska to tilting more to Greenwich meridian for leads located away from the coastline.

\subsection{Monthly 9-Year-Average Lead Orientation}

In Section 5.1, we presented the spatial distribution of lead orientations that does not contain information about the spatio-temporal variability from 2002 to 2011. In this section, we show monthly averages that represent the temporal variability of all years (Figure 11). The standard deviation is again 
shown as line thickness and white dots on top of a cell indicate that the shown lead orientations belong to a statistically significant non-random distribution. We use the Monte Carlo approach as presented in Section 5.1 to test if lead orientations have a random distribution.

Figure 11. Monthly averaged lead orientation from 2002 to 2011. Grey colors display the number of leads while the width of a lead measures the standard deviation of all lead orientations in a $200 \mathrm{~km} \times 200 \mathrm{~km}$ cell. White dots on top of a line indicate that the lead orientations are not randomly distributed on a confidence level of $99 \%$.

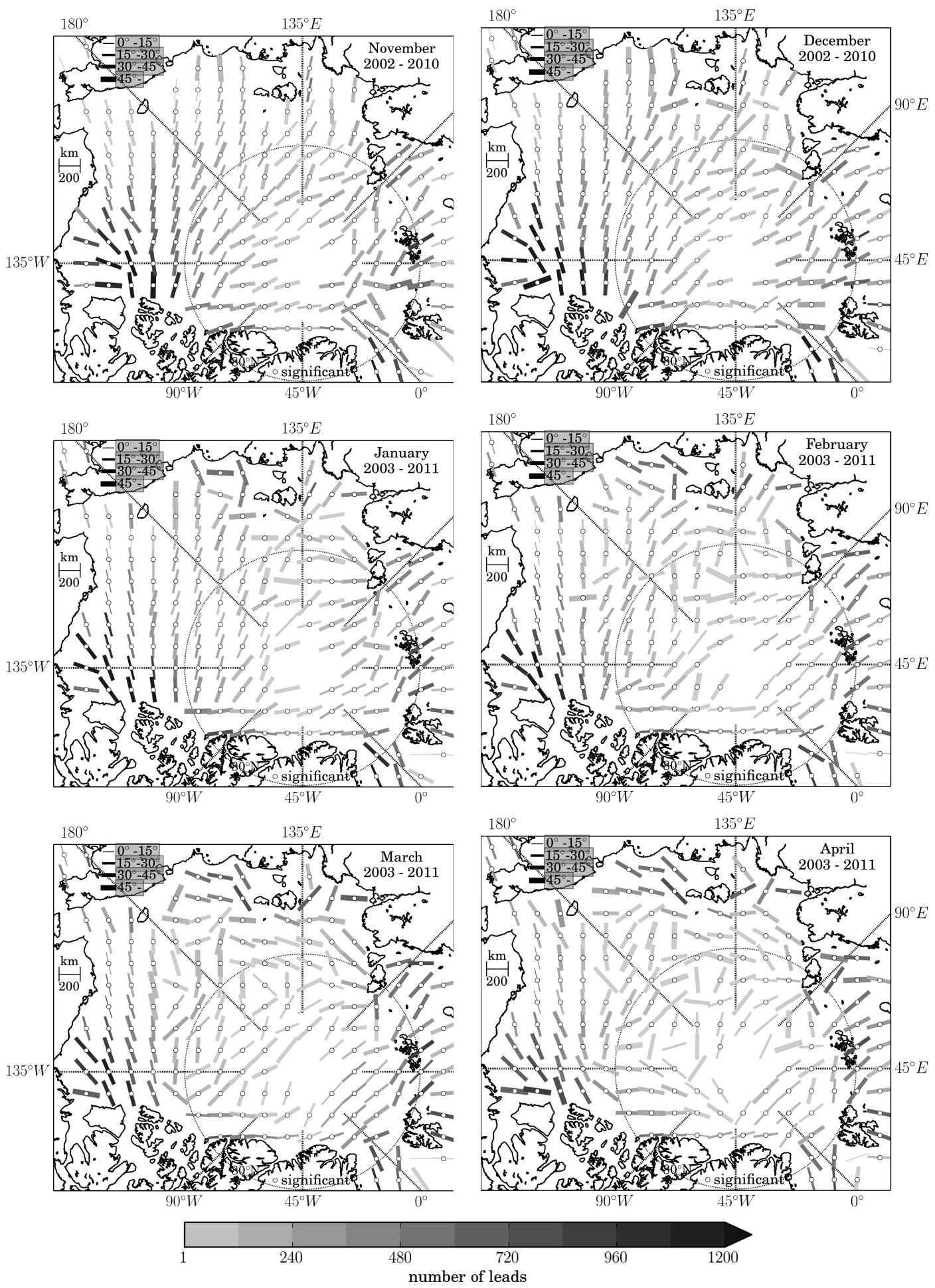


The overall spatial pattern is not that smooth for every month, lead orientations vary especially in the central Arctic and for months from February to April as compared to the average over all years (Figure 10). More leads are detected at the beginning of winter with lead occurrence always peaking in the Beaufort Sea. For the Beaufort Sea, we observe that the amount of leads that are orientated perpendicular to the coastline of Alaska decreases with ongoing winter. In the Fram Strait exit region, leads are orientated almost parallel to Greenwich meridian in November, while the leads get more and more orientated perpendicular to Greenwich meridian as the winter season advances. Leads orientated parallel to the coast of Siberia build up from December/January to April. In the central Arctic, leads perpendicular to Greenwich meridian tend to tilt parallel to the coastline of Alaska with ongoing season. Contemporary to this directional tilt the number of detected leads declines like in every region of the Arctic.

\subsection{Time Series of Lead Orientation in the Fram Strait and in the Beaufort Sea}

In order to get an idea of the monthly variability, we show the lead orientation for two sub regions of the Fram Strait and the Beaufort Sea from 2002 to 2011 (Figure 12). Like in Figure 7, we depict the lead orientation as angles. The graph of the Fram Strait is depicted in black, the graph of the Beaufort Sea in gray, consistent to the gray and black boxes that mark the corresponding sub regions in Figure 10. Both graphs are based on monthly averages.

Figure 12. Time series of monthly averaged lead orientations in sub regions of the Beaufort Sea (black dashed) and the Fram Strait (gray) from 2002 to 2011 . Both, $0^{\circ}$ and $180^{\circ}$, are orientated parallel to Greenwich meridian. The precise location of the two sub regions is indicated by boxes in Figure 10. The lines on the left hand side illustrate the corresponding lead orientation.

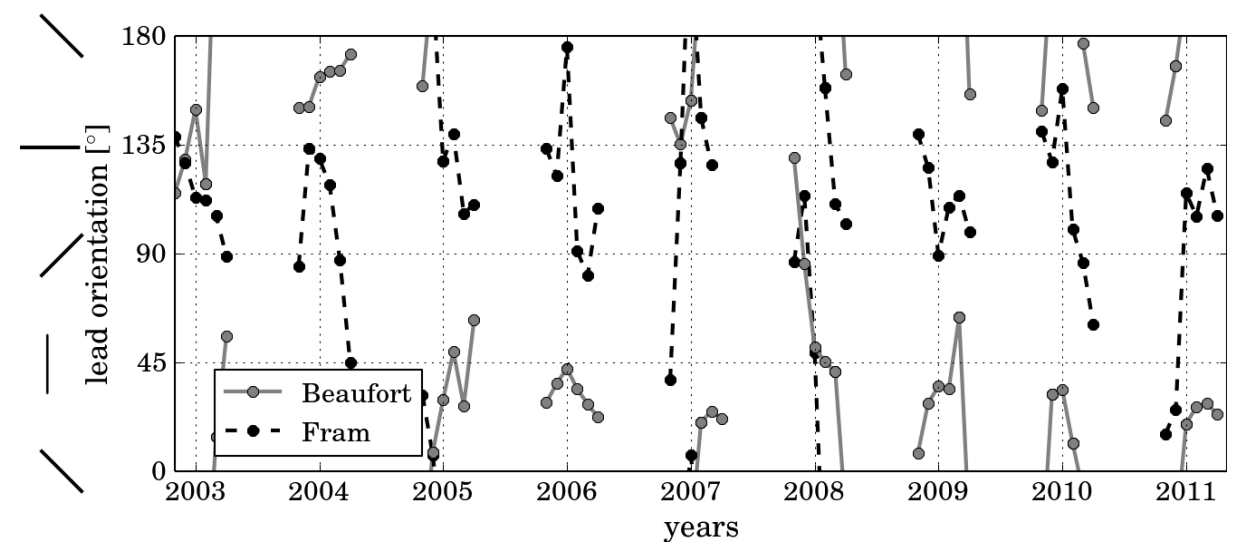

The lead orientations in both regions are largely distributed. The most prominent exceptions from the large spread in lead orientation are the years 2004 and 2006 in the Beaufort Sea. In the Fram Strait, leads are orientated more parallel to Greenwich meridian in the beginning of a season, but become more perpendicular to Greenwich meridian during the season. We observe that leads get more orientated parallel to Greenwich meridian in the Beaufort Sea as the season advances. Still, all of the tendencies are dominated by large fluctuations within the seasons. 


\section{Discussion}

In Section 6.1, we discuss uncertainty and limitations of the developed approach to derive the lead orientation. A short comparison of our results to two reference studies follows in Section 6.2. In Section 6.3, we comment on the patterns of obtained lead orientations although we cannot provide a complete explanation.

\subsection{Uncertainty and Limitations}

The Hough transform by Galambos et al. [8] does not include an internal uncertainty estimate for a detected line. Therefore, we cannot provide an individual uncertainty for every detected lead. In order to estimate the uncertainty we distinguish between two types of lead detections: a lead that results from only one detection and a lead that results from at least two lead detections, which are clustered to one resulting lead. For every detected lead we provide the C-score (Equation (4) introduced in Section 3.4) as a first measure of uncertainty. For every clustered lead orientation resulting from at least two lead detections we provide an uncertainty angle. We define the uncertainty angle as the angular standard deviation ( $c f$. Section 4.2) of detected leads which are clustered to one lead. To give an example, the uncertainty angles for the three clustered leads in the zoom of Figure 2 are $2.4^{\circ}, 1.0^{\circ}$, and $5.4^{\circ}$ from top to bottom.

In Table 2 we provide three average uncertainty estimates for the Fram Strait and the Beaufort Sea obtained on two different days. The root mean square deviation is calculated between reference lead orientations and corresponding lead orientations detected by the Hough transform. The root mean square deviation is lowest in the Fram Strait (F) compared to higher values in the Beaufort Sea (B1, B2). The mean uncertainty angle is lowest in the Fram Strait (F) and highest in the Beaufort Sea (B2) on 14 November 2004. A possible explanation for the differences is that leads are located closer to neighboring leads in the Beaufort Sea (B2). Furthermore, almost the entire directional range is covered with entries (Figure 8) and the number of leads is twice as high as Beaufort Sea (B2) compared to the Fram Strait (F).

Table 2. Root mean square deviation, uncertainty angle, and C-score calculated for three regions: Fram Strait (F) on 14 March 2011 (Figure 5), Beaufort Sea (B1) on the same date (map not shown), and Beaufort Sea (B2) on 14 November 2004 (Figure 6).

\begin{tabular}{lrrr}
\hline & \multicolumn{3}{c}{ Region } \\
& F & B1 & B2 \\
\hline Root mean square deviation $\left[^{\circ}\right]$ & 8.5 & 13.5 & 12.9 \\
Mean uncertainty angle $\left[^{\circ}\right]$ & 2.9 & 7.2 & 14.9 \\
Mean C-score & 0.94 & 0.94 & 0.88 \\
\hline
\end{tabular}

Another source of uncertainty is the impact of a quantization effect. By quantization effect we mean that the shorter the lead length is, the less options the Hough transform has to fit a line through this lead. The quantization effect would become more pronounced if we detected for example leads with a length of only two pixels. The only possible lead orientations would be $0^{\circ}, 45^{\circ}, 90^{\circ}$, and $135^{\circ}$. We 
set the minimal line length of a lead to five pixels resulting in considerably more options for a possible lead orientation. The clustering of leads reduces the quantization effect by averaging leads with slightly different orientations. Nonetheless, the peak between $130^{\circ}$ and $140^{\circ}$ in the distribution of the Hough angles in comparison with the reference angles could partly be due to the quantization effect (Figure 7). However, the bin width should be $30^{\circ}$ instead of $10^{\circ}$ following Sturges rule given a sample size of around 35 angles.

Uncertainty is also caused by the tendency that the daily standard deviation of lead orientations is lower in the Fram Strait than in the Beaufort Sea. We assess this observation as a hint for more unidirectional lead orientations in the Fram Strait. On some days the Hough transform detects unidirectionally distributed leads more accurately than isotropically distributed leads. Thus, lead orientations might be more robust in the Fram Strait, as well as north of Siberia, and the central Arctic than in the Beaufort Sea, where leads are more isotropically distributed and less certainly detectable.

A limitation of our approach is the resolution of the AMSR-E based lead concentration. The applied algorithm to infer the lead concentration detects leads down to $3 \mathrm{~km}$ in width smaller than the AMSR-E pixel size of $6.25 \mathrm{~km} \times 6.25 \mathrm{~km}$ by using an edge detecting filter [9]. Due to both (A and B) 89 $\mathrm{GHz}$ scans available, AMSR2 allows to produce brightness temperature maps with a pixel size of $3.125 \mathrm{~km} \times 3.125 \mathrm{~km}$ [26]. We did not yet apply the Hough transform on AMSR2 based lead concentration. Nonetheless, the AMSR-E sensor is able to detect fragments of leads narrower than $3 \mathrm{~km}$ in width. The structure of the fragments in the sea ice cover is often none line-like and to infer a lead orientation from these geometrical structures is not robust. In order to get rid of single pixels originating from unresolved leads, we apply a morphological hit-or-miss transform that erases all single pixels in a binary lead concentration map. Furthermore, we are able to isolate lead structures from unresolved lead fragments by applying the Hough transform on the lead concentration.

As mentioned in Section 3.5, we cannot find a combination of one threshold parameter and one line length that accurately fits for every day. This finding is a consequence of the spatial (between regions) and temporal (between days) variability. While on certain days longer leads prevail, a longer minimal line length combined with a lower threshold value accurately depicts the longer lead patterns. On other days, shorter leads prevail that are accurately depicted by a shorter minimal line length combined with a higher threshold value.

We do not analyze the distribution of lead length for two reasons: Firstly, the Hough transform tends to underestimate the lead length as visible in Figure 5. Secondly, the lead concentration does not resolve small lead structures. Thus, we cannot tell from the lead concentration alone where a lead begins or ends.

\subsection{Comparison to Former Studies}

Reference publications covering the lead orientation in the Arctic are rare. We compare our results qualitatively to the results of Miles and Barry [4] and Lindsay and Rothrock [3]. Lindsay and Rothrock show a map of the Fram Strait for 25 February 1989 and 1 March 1989 with leads orientated perpendicular to Greenwich meridian [3]. We often observe the same lead orientation in the Fram Strait.

Miles and Barry [4] show maps of mean lead orientation in the Beaufort Sea for winters from 1979 to 1985. When we compare their pattern of lead orientation to mean lead orientations from 2003 to 2008 
(Figure 13), we observe a shift away from the coastline. The mean lead orientation of Miles and Barry [4] remains more perpendicular to the coastline whereas the recent mean lead orientation is tilted parallel to the coastline. Near the coastline the lead orientation is similarly orientated. We do not know at this point the reasons that explain these differences between the different years. To find out if the differences can be explained by the spinning up of the Beaufort gyre during the 2000s and an increased ice deformation is subject to future work [27,28]. As already pointed out in the previous section, our method may not be that robust in the Beaufort Sea due to the more isotropically distributed leads compared to other regions of the Arctic.

Figure 13. Average lead orientation from 2003 to 2008 in an identically rotated map as the map in Figure 6 of Miles and Barry [4]. Grey colors display the number of leads while the width of a lead measures the standard deviation of all lead orientations in a $200 \mathrm{~km} \times 200 \mathrm{~km}$ cell. White dots on top of a mean lead orientation indicate a statistically significant preferred orientation [4].

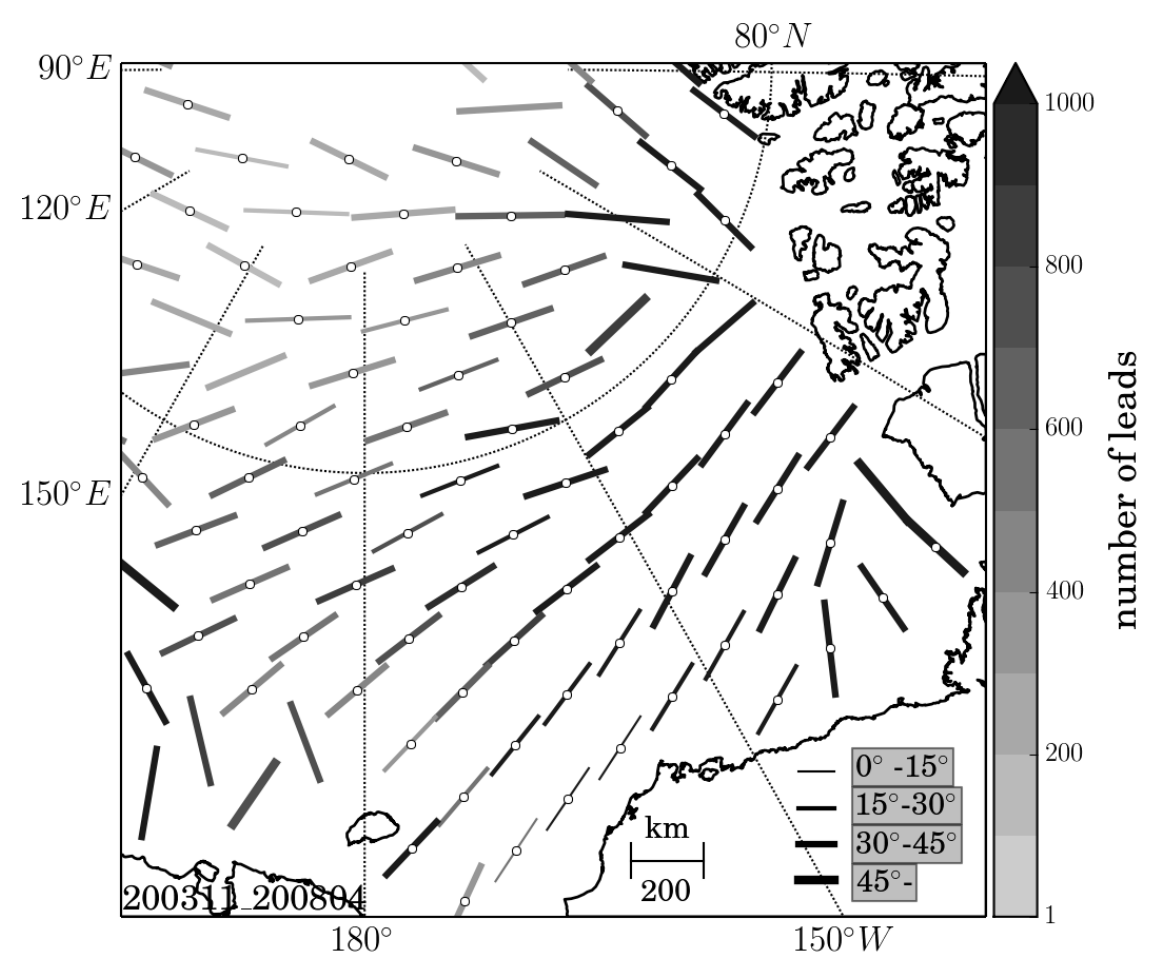

Miles and Barry [4] found statistically significant preferred lead orientations in a cell with the strength of preference $R$ [4,23], which is defined as:

$$
R=N^{-1} \sqrt{\bar{X}^{2}+\bar{Y}^{2}}
$$

The strength of preference $R$ ranges from 0 for random orientations to 1 for only one orientation occurring in a cell [4]. Miles and Barry [4] state that a cell has a statistically significant preferred lead orientation for $R \gtrsim 0.25$ and $N \geq 60$ with $N$ being the number of observations. We find statistically significant preferred lead orientations in 77 out of 92 grid cells in comparison to 38 out of 63 grid cells for Miles and Barry [4]. The rise of about $30 \%$ in the number of cells with statistically significant preferred lead orientation is partly due to fewer randomly orientated lead orientations north of $80^{\circ} \mathrm{N}$ and in the Bering Sea entrance region in our study. 
We did not use the test by Miles and Barry [4] in previous sections to check for a statistically significant preferred mean lead orientation, because we think it is more suitable to check if lead orientations are statistically significantly non-randomly distributed in a cell. Furthermore, Miles and Barry [4] do not account with their test for a potential secondary preferred mean orientation or other shapes of the distribution.

\subsection{Distribution of Lead Orientation}

The smooth spatial pattern indicates a spatial coherence of lead orientations during the entire winter season (Figures 10 and 11). One reason for this spatial coherence might be orographically forced, because leads tend to be orientated perpendicular to a coastline. Leads are only orientated parallel to the coastline north of Greenland, north of Siberia, and partly north of Alaska. The coast-parallel lead structure north of Greenland and partly north of the Canadian Archipelago is probably due to thicker multi-year ice. In these regions, we observe that sea ice tends to break up parallel to the sea ice thickness gradient and to the shoreline. The situation north of Siberia differs: In the beginning of winter, leads are perpendicular to the shoreline but they tilt parallel to the shoreline when the fast-ice develops north of Siberia in the Barents sea, Kara sea [29], and Laptev sea (Figure 11). In the Fram Strait exit region, leads orientated perpendicular to Greenwich meridian can be interpreted as traces of blocking events or arches [30] from January to April (Figure 11).

Leads are statistically significantly non-randomly orientated almost everywhere in Arctic winters when averaged over all years (Figures 10 and 11). The few cases in which a cell with a random distribution of lead orientations occurs may partly be due to a small number of observations in that cell. The existence of a statistically significant non-random distribution of lead orientations in many cells is also connected to both the spatial dimensions of a cell and to the time period over which it is averaged. Lowering the time period and decreasing the cell dimensions results in fewer leads per cell. This causes more randomly orientated leads even in cells with many lead observations like in the Beaufort Sea.

Figure 14. Histogram of lead orientations in sub regions of the Beaufort Sea (gray) and the Fram Strait (black solid line) from 2002 to 2011. The precise locations of the two sub regions are shown in Figure 10.

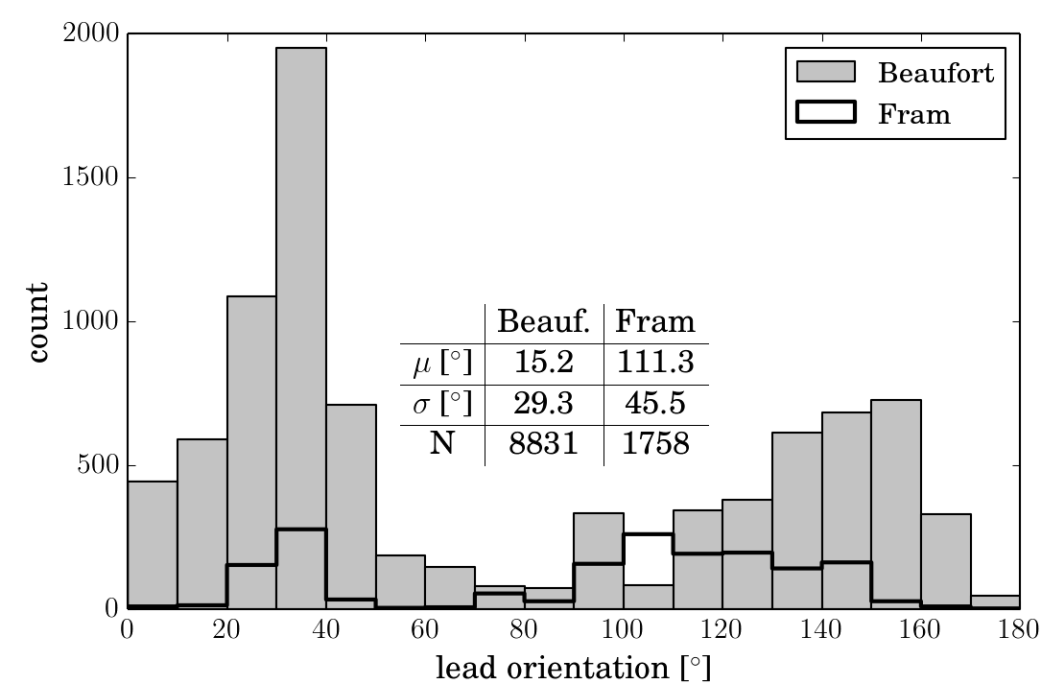


As an example, we show the distribution of lead orientations for two sub regions (Figure 14) from 2002 to 2011 in order to demonstrate that we obtain statistical-significant non-random lead distributions as indicated in Figures 10 and 11. The distribution of lead orientations in the Beaufort Sea peaks in the bin from $30^{\circ}$ to $40^{\circ}$ and shows a secondary peak in the bin from $150^{\circ}$ to $160^{\circ}$. The distribution of lead orientations in the Fram Strait peaks in the bin from $90^{\circ}$ to $100^{\circ}$ and shows a secondary peak in the bin from $30^{\circ}$ to $40^{\circ}$. The lead orientations in the Fram Strait are much more broadly distributed which is consistent with the larger standard deviation. The distributions show in both regions visually no similarity to a random uniform distribution. Consistently, the $\chi^{2}$-test applied within a Monte Carlo simulation like in Section 4.2 returns very low probabilities for randomly distributed lead orientations. The very low probabilities emphasize that lead orientations are statistically significantly non-randomly distributed.

When we look at the average spatial pattern of lead orientation from 2002 to 2011 (Figure 10), one can ask: What are the prevailing processes that cause this pattern? An in-depth answer to this question would clearly require a comparison to additional observations, like surface wind or sea ice drift. Finding possible connections will be subject of future work.

\section{Conclusions}

The presented algorithm successfully determines the positions and orientations of leads larger than $3 \mathrm{~km}$ in width. The algorithm is not necessarily applicable for detecting the length of a lead, but for detecting the orientation of a lead. Even though the algorithm does not detect every lead, the algorithm infers the mean lead orientation and a similar distribution of lead orientations compared to manually obtained lead orientations. An extensive validation of detected lead orientations with Advanced Synthetic Aperture Radar scenes reveals a good agreement for large leads.

Applying the algorithm to Advanced Microwave Scanning Radiometer for EOS based lead concentration for a period from 2002 to 2011 we find that the spatial lead distribution tends to be coherent over large distances. Leads are on average orientated parallel to their neighboring leads. On the monthly timescale the lead orientation varies regionally more strongly than on the yearly or multi-yearly timescale. A statistical-significant non-random distribution of lead orientations prevails in most regions of the Arctic in winters from 2002 to 2011. This indicates that on the scale typical for Arctic-wide or global models sea ice should be described with an anisotropic rheology rather than an isotopic rheology.

\section{Acknowledgements}

We thank all researchers involved in the improvement of this publication. Special thanks go to Dirk Notz for very helpful discussions concerning this article. We gratefully acknowledge the Ice Route Optimization - 2 (IRO-2) project for financial support. The IRO-2 project is funded by the German Federal Ministry of Economics and Technology from October 2011 to September 2014 in the framework of the program "Shipping and ocean engineering for the $21^{\text {st }}$ century". We thank ESA for supplying the ASAR WSM images and JAXA as well as National Snow and Ice Data Center (NSIDC) for supplying the AMSR-E brightness temperatures. 


\section{Author Contributions}

Lars Kaleschke developed the main idea that led to this paper as part of a work package within the IRO-2 project. David Bröhan developed the methods, performed the analysis, and wrote the paper as part of his $\mathrm{PhD}$ under the supervision of Lars Kaleschke.

\section{Conflicts of Interest}

The authors declare no conflicts of interest.

\section{References}

1. Kwok, R. Deformation of the Arctic Ocean Sea Ice Cover: November 1996 through April 1997. In Scaling Laws in Ice Mechanics and Dynamics; Dempsey, J., Shen, H.H., Eds.; Kluwer Academic: Dordrecht, The Netherlands, 2001; pp. 315-322.

2. Hutchings, J.; Heil, P.; Hibler, W. Modeling linear kinematic features in sea ice. Mon. Wea. Rev. 2005, 133, 3481-3497.

3. Lindsay, R.; Rothrock, D. Arctic sea-ice leads from Advanced Very High-Resolution Radiometer images. J. Geophys. Res.: Oceans 1995, 100, 4533-4544.

4. Miles, M.W.; Barry, R.G. A 5-year satellite climatology of winter sea ice leads in the western Arctic. J. Geophys. Res. 1998, 103, 21,723-21,734.

5. Zakhvatkina, N.; Alexandrov, V.; Johannessen, O.; Sandven, S.; Frolov, I. Classification of sea ice types in ENVISAT Synthetic Aperture Radar images. IEEE Trans. Geosci. Remote Sens. 2013, $51,2587-2600$.

6. Fetterer, F.; Holyer, R. A Hough Transform Technique for Extracting Lead Features from Sea Ice Imagery; Naval Ocean R\&D Activity: SSC, MS, USA, 1989; pp. 1125-1128.

7. Bandfield, J. Skeletal modeling of ice leads. IEEE Trans. Geosci. Remote Sens. 1992, 30, 918-923.

8. Galambos, C.; Matas, J.; Kittler, J. Progressive Probabilistic Hough Transform for Line Detection. In Proceedings of 1999 IEEE Computer Society Conference on Computer Vision and Pattern Recognition, Ft. Collins, CO, USA, 23-25 June 1999; pp. 554-560.

9. Röhrs, J.; Kaleschke, L.; Bröhan, D.; Siligam, P. An algorithm to detect sea ice leads using AMSR-E passive microwave imagery. Cryosphere 2012, 6, 365-365.

10. Tsamados, M.; Feltham, D.L.; Wilchinsky, A.V. Impact of a new anisotropic rheology on simulations of Arctic sea ice. J. Geophys. Res.: Oceans 2013, 118, 91-107.

11. Girard, L.; Weiss, J.; Molines, J.M.; Barnier, B.; Bouillon, S. Evaluation of high-resolution sea ice models on the basis of statistical and scaling properties of Arctic sea ice drift and deformation. $J$. Geophys. Res. 2009, 114, C08015.

12. Wilchinsky, A.; Feltham, D. A continuum anisotropic model of sea ice dynamics. Proc. R. Soc. Lond. A 2004, 460, 2105-2140.

13. Girard, L.; Bouillon, S.; Weiss, J.; Amitrano, D.; Fichefet, T. A new modeling framework for sea ice mechanics based on elasto-brittle rheology. Ann. Glaciol. 2011, 52, 123-132. 
14. Sulsky, D.; Peterson, K. Toward a new elasticdecohesive model of Arctic sea ice. Physica D 2011, 240, 1674-1683.

15. Wilchinsky, A.; Feltham, D. Rheology of Discrete Failure Regimes of Anisotropic Sea Ice. $J$. Phys. Oceanogr. 2012, 42, 1065-1082.

16. Cavalieri, D. J.; Markus, T.; Comiso, J. AMSRE/Aqua Daily L3 12.5 km Brightness Temperature, Sea Ice Concentration, \& Snow Depth Polar Grids Ver. 2; NASA DAAC at the National Snow and Ice Data Center: Boulder, CO, USA, 2003.

17. Cavalieri, D. J.; Markus, T.; Comiso, J. AMSRE/Aqua Daily L3 6.25 km 89 GHz Brightness Temperature Polar Grids Ver. 2; NASA DAAC at the National Snow and Ice Data Center: Boulder, CO, USA, 2004.

18. Kaleschke, L.; Heygster, G.; Lüpkes, C.; Bochert, A.; Hartmann, J.; Haarpaintner, J.; Vihma, T. SSM/I sea ice remote sensing for mesoscale ocean-atmosphere interaction analysis: Ice and icebergs. Can. J. Remote Sens. 2001, 27, 526-537.

19. Spreen, G.; Kaleschke, L.; Heygster, G. Sea ice remote sensing using AMSR-E 89-GHz channels. J. Geophys. Res. 2008, 113, C02S03.

20. ICDC. AMSR-E Lead Area Fraction for the Arctic; [2002-2011]; Integrated Climate Date Center, University of Hamburg: Hamburg, Germany, 2013.

21. Hough, P.V.C. Methods and Means for Recognizing Complex Patterns. US 3069654, 12, 1962.

22. Duda, R.O.; Hart, P.E. Use of the Hough Transformation to detect lines and curves in pictures. Commun. ACM 1972, 15, 11-15.

23. Mardia, K.; Jupp, P. Directional Statistics; Wiley: Chichester, UK, 2000.

24. Olson, E. On Computing the Average Orientation of Vectors and Lines. In Proceedings of 2011 IEEE International Conference on Robotics and Automation (ICRA), Shanghai, China, 9-13 May 2011; pp. 3869-3874.

25. Scott, D.W. Multivariate Density Estimation: Theory, Practice, and Visualization, 1st ed.; Wiley Series in Probability and Statistics; Wiley: New York, USA 1992.

26. Beitsch, A.; Kaleschke, L. The February 2013 Arctic Sea Ice Fracture in the Beaufort Sea-A case study for two different AMSR2 sea ice concentration algorithms. Remote Sens. 2013, submitted.

27. Giles, K.; Laxon, S.; Ridout, A.; Wingham, D.; Bacon, S. Western Arctic Ocean freshwater storage increased by wind-driven spin-up of the Beaufort Gyre. Nature Geosci. 2012, 5, 194-197.

28. Morison, J.; Kwok, R.; Peralta-Ferriz, C.; Alkire, M.; Rigor, I.; Andersen, R.; Steele, M. Changing Arctic Ocean freshwater pathways. Nature 2012, 481, 66-70.

29. Olason, E.O. Dynamical modelling of Kara Sea Land-Fast Ice. Ph.D. Thesis, University of Hamburg, Hamburg, Germany, 2012.

30. Kwok, R.; Pedersen, L.T.; Gudmandsen, P.; Pang, S.S. Large sea ice outflow into the Nares Strait in 2007. Geophys. Res. Lett. 2010, 37, L03502. 


\section{Appendix}

Table A1. 50 Hough input parameter combinations (threshold value (thres) and minimal line length (mll)) ranked according to the $\mathrm{C}$-score for a test period covering the whole Arctic and lasting from February to April 2011.

\begin{tabular}{lccccccccccccccccc} 
rank & 1 & 2 & 3 & 4 & 5 & 6 & 7 & 8 & 9 & 10 & 11 & 12 & 13 & 14 & 15 & 16 & 17 \\
thres & 38 & 31 & 15 & 48 & 40 & 36 & 35 & 29 & 26 & 25 & 23 & 22 & 20 & 17 & 13 & 50 & 48 \\
mll & 6 & 5 & 5 & 5 & 5 & 8 & 6 & 6 & 5 & 6 & 5 & 5 & 5 & 5 & 6 & 5 & 8 \\
\hline rank & 18 & 19 & 20 & 21 & 22 & 23 & 24 & 25 & 26 & 27 & 28 & 29 & 30 & 31 & 32 & 33 & 34 \\
thres & 47 & 46 & 45 & 44 & 43 & 42 & 42 & 41 & 37 & 37 & 37 & 34 & 33 & 29 & 28 & 27 & 26 \\
mll & 7 & 6 & 7 & 5 & 5 & 8 & 6 & 6 & 7 & 6 & 5 & 6 & 5 & 5 & 8 & 5 & 6 \\
\hline rank & 35 & 36 & 37 & 38 & 39 & 40 & 41 & 42 & 43 & 44 & 45 & 46 & 47 & 48 & 49 & 50 & \\
thres & 25 & 24 & 22 & 21 & 13 & 11 & 10 & 50 & 48 & 47 & 47 & 46 & 43 & 42 & 41 & 41 \\
mll & 5 & 6 & 6 & 5 & 5 & 5 & 6 & 6 & 6 & 6 & 5 & 5 & 8 & 5 & 7 & 5
\end{tabular}

Listing 1. Source code written in the programmin language python of a function to calculate the angular standard deviation: The function needs as input a set of orientations $\theta_{i}$ and their corresponding mean value $\bar{\theta}$.

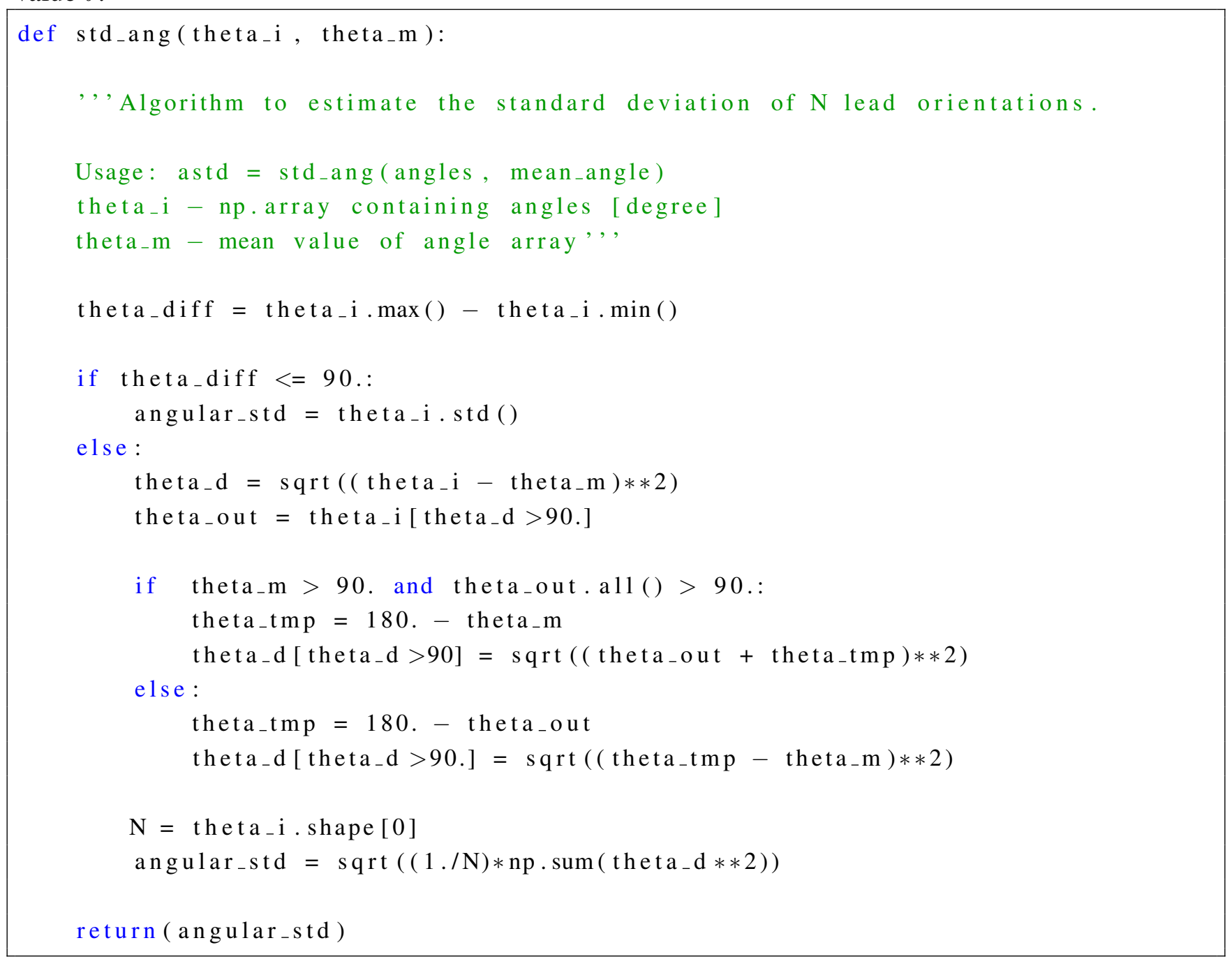

(c) 2014 by the authors; licensee MDPI, Basel, Switzerland. This article is an open access article distributed under the terms and conditions of the Creative Commons Attribution license (http://creativecommons.org/licenses/by/3.0/). 